\title{
El cuerpo como principio de libertad en Spinoza
}

\author{
The body as principle of freedom in Spinoza
}

\author{
IVÁN RAMÓN RODRÍGUEZ BENAVIDES*
}

\begin{abstract}
Resumen: Con este artículo exponemos algunos alcances políticos del pensamiento de Spinoza en torno al cuerpo. Consideramos que Spinoza es uno de los primeros filósofos de la modernidad que se ocupa de pensar el cuerpo en la política, para hacerlo rompe con la presunción clásica de la oposición entre cuerpo y alma como dos sustancias que conforman al hombre, de las cuales la parte esencial sería el alma. En cambio, para Spinoza el cuerpo es tan constitutivo de lo humano como el alma, «alma y cuerpo son una y la misma cosa», así apreciamos, en parte, la novedad de su pensamiento.
\end{abstract}

Palabras clave: Spinoza, cuerpo, política, composición, poder.

\begin{abstract}
With this article, we present some political scope of Spinoza's thought about the body. We believe that Spinoza is one of the first philosophers of modernity that deals with thinking the body in politics, because of that breaks up with the classical assumption of the opposition between body and soul as two substances that make the man, where the soul is the part essential. However, for Spinoza the body is so constitutive of the human as the soul, «soul and body are one and the same thing», thereby we can appreciate, in part, the novelty of his thinking.
\end{abstract}

Keywords: Spinoza, body, politics, composition, power.

En la explicación del mundo como un orden necesario, además del cambio en la concepción de Dios, también se produce un cambio en la concepción del hombre, y ese cambio le permitirá a Spinoza dar cuenta definitiva de los prejuicios, especialmente de aquellos que causa la imaginación, tal como lo había planteado en el Apéndice de la primera parte de la Ética. Este planteamiento nos permitirá entender por qué el hombre ha visto reducido su conatus o su libertad por el miedo que le han infundido tanto la religión como la política. En la segunda parte de la Ética, "De la Naturaleza y Origen del Alma", Spinoza se concentra en exponer ese cambio que se lleva a cabo en la concepción antropológica.

Fecha de recepción: 09/06/2016. Fecha de aceptación: 06/09/2016.

* Profesor de la Facultad de Filosofía y Humanidades de la Universidad de La Salle (Bogotá). Me dedicó a la investigación política, especialmente los problemas fundacionales del Estado y los modelos de Democracia, además también investigo en torno a los problemas de la comprensión desde la Hermenéutica. Rodríguez, Iván (2015): Las virtudes cívicas, una contribución a la Paz en Perspectivas Multidimensionales para la Paz, Ibañez, Bogotá D.C. Rodríguez Iván (2014): Contribución a las consideraciones en torno al perdón en Política en Cartografías de la Paz, una mirada crítica al territorio, Ediciones Unisalle, Bogotá D.C. ivrodriguez@unisalle. edu.coivanra2@yahoo.fr 
En primer lugar es pertinente destacar que Spinoza es uno de los primeros filósofos de la modernidad que se ocupa de pensar el cuerpo en la política, y que para hacerlo rompe con la presunción clásica de la oposición entre cuerpo y alma como dos sustancias que constituyen al hombre de las cuales la parte esencial sería el alma. En cambio, para Spinoza el cuerpo y el alma son dos modos del mismo cuerpo, "alma y cuerpo son una y la misma cosa"1. Esta concepción de la unidad cuerpo y alma establece además una relación de concomitancia, a saber, lo que siente el cuerpo es concomitante a lo que piensa el alma y lo que piensa el alma es concomitante a lo que siente el cuerpo, por lo tanto "un cambio en el estado de mi cuerpo no causará un cambio en el estado de mi mente; es ya un cambio en mi mente"2.

Spinoza resuelve de otra manera la discusión que había planteado Descartes respecto a la comunicación entre el alma y el cuerpo, puesto que cada atributo sigue en la expresión de sus modos el mismo orden y necesidad, pero ningún atributo se comunica con los otros atributos, como lo hemos explicado anteriormente. No es pertinente hablar, por lo tanto, de una acción causal entre modos de distintos atributos, puesto que "el orden y la conexión de las ideas es el mismo que el orden y la conexión de las cosas"3.

De ahí que la comunicación sólo se pueda dar entre modos del mismo atributo: el alma con los modos del pensamiento, esto es las ideas, y el cuerpo con los modos de la extensión, es decir los cuerpos ${ }^{4}$. En este sentido, no se trata de una comunicación entre atributos, sino del orden y conexión idénticos de cada uno de ellos en la expresión de sus respectivos modos. Así se establece una distinción cualitativa entre los atributos, y no una distinción cuantitativa, y por ende no cuantificable. Es decir, Spinoza considera que alma y cuerpo son dos aspectos de una realidad única: el aspecto extenso y el aspecto racional, el uno accesible por medio de los sentidos y el otro accesible por medio del entendimiento. De esta manera vuelve a insistir en que sólo existe una única sustancia, pero que el entendimiento infinito entiende esa única sustancia bien bajo el atributo extensión o bien bajo el atributo pensamiento. Además, esto quiere decir, como lo señala en el escolio b de la proposición 7 de la segunda parte, que Dios es la causa de todas las cosas, sean ellas modos del atributo pensamiento, o sean modos del atributo extensión, y que el orden causal en el seno de cada atributo debe ser explicado por ese mismo atributo.

Ahora bien, de acuerdo con esta explicación, podemos preguntarnos ¿Qué es el hombre en el orden natural? Y la respuesta a este interrogante la encontramos en el Corolario de la Proposición 13: "el hombre consta de alma y cuerpo, y el cuerpo humano existe tal como

1 Smith, S., Spinoza y el libro de la vida. Libertad y Redención en la Ética, Madrid, Biblioteca Nueva, 2007, p. 102.

2 Op. Cit. p. 109.

3 EIIP7.

4 Algunos comentaristas de Spinoza suelen asimilar esta explicación con el planteamiento de la "armonía preestablecida" y el consiguiente "paralelismo" de Leibniz. Sin embargo, esa analogía no es muy acertada, puesto que si bien hay una distinción real entre los atributos, y por lo tanto entre los modos de esos atributos, esa distinción no es sustancial, no es entre dos cosas distintas, sino entre dos modalidades de una misma cosa, de una misma sustancia. Para Deleuze, aunque Spinoza también se refiere a la correspondencia, intenta ir más allá para explicarla, puesto que muestra no sólo un mismo orden, sino una isonomía, esto es, una igualdad de principios entre series autónomas e independientes. Deleuze elabora todas las explicaciones que pueden surgir de los matices que introduce Spinoza con su comprensión del paralelismo en el capítulo VI de su libro "Spinoza y el problema de la expresión". 
lo sentimos"5. En esa constitución, el cuerpo es concebido como el objeto del alma, con lo cual Spinoza vuelve a insistir que el orden necesario con que sucede algo en el cuerpo es el mismo que el orden necesario con el que se conoce en el alma, pues, como queda claro desde el axioma 6 de la primera parte: "la idea verdadera debe concordar con su objeto ideado". De tal manera que, aun si no puede hablarse de comunicación, ni por consiguiente de causalidad entre modos de atributos distintos, se presenta una correlación mediante el paralelismo que se da entre dichos modos. Así, "alma y cuerpo son una y la misma cosa"6. Empleando la explicación de Deleuze podríamos decir que lo que cambia es el punto de vista, posición que queda explícita en la proposición 2 de la Tercera parte cuando Spinoza sostiene que "ni el cuerpo puede determinar al alma a pensar, ni el alma puede determinar al cuerpo al movimiento ni al reposo, ni a alguna otra cosa"

De esta manera todo lo que sucede en el cuerpo tiene una correspondencia 'mental', es decir, que todo lo que sucede en el cuerpo es comprensible. Sin embargo, que sea comprensible no significa que nosotros lo comprendamos de hecho. Lo que sí es claro para nosotros es su existencia, como lo señalaba la cita del Corolario de la Proposición 13 del libro 2: "el cuerpo humano existe tal como lo sentimos". De esta manera Spinoza se aparta de la duda racionalista que desconfía de los sentidos, hasta llegar incluso a poner en duda su propia existencia bajo la metáfora del "cerebro suspendido", para Spinoza la certeza de la existencia del cuerpo es indudable. Aunque esto no significa que tengamos una comprensión adecuada del cuerpo, que lo entendamos de manera adecuada, pues si el alma es la idea del cuerpo, no es la idea que nosotros tenemos de nuestro cuerpo, sino la estructura racional que lo articula como un juego de fuerzas. Por eso el alma es la idea 'que Dios tiene' del cuerpo, es decir, la estructura racional del mismo. Así, tal como lo explica Smith:

un cambio en el estado de mi cuerpo no causará un cambio en el estado de mi mente; es ya un cambio en mi mente. El mundo de los cuerpos y el mundo mental no son dos mundos diferentes, sino el mismo mundo, aunque descrito desde dos puntos de vista diferentes?

Además, Spinoza sostiene que el alma en tanto idea sólo puede ser idea de una cosa existente, y sólo así puede pensarse que es idea del cuerpo en tanto que éste existe, aunque la cosa existente, el cuerpo, no existe de forma necesaria, sino de forma contingente, de tal manera que no justifica su existencia por sí mismo, sino por otro, que es su causa próxima, y en último término por Dios, que es su causa última.

Ahora bien, otra característica de un cuerpo y por supuesto del hombre es su singularidad, que hace que los hombres sean diferentes. Al respecto Spinoza sostiene que la singularidad está dada por la finitud y la existencia limitada. Además, señala que el actuar con un propósito común, de tal manera que se persiga un mismo efecto, también genera individualidad ${ }^{8}$, noción que, en primer lugar, servirá para explicar el cuerpo humano como conformidad de

5 EIIP13cor.

6 Spinoza y el libro de la vida, Op. Cit. p. 102.

7 Op. Cit.p. 109.

8 Sin embargo, Spinoza terminará de explicar su concepción de la diferencia entre los hombres desde la potencia, pero de ello nos ocuparemos más adelante. 
varios cuerpos que buscan un mismo efecto, pero que, en segundo lugar, en el campo político también tendrá una aplicación. "Y si varios individuos concurren a una misma acción de tal manera que todos a la vez sean causa de un solo efecto... los considero a todos ellos como una cosa singular"9.

En la primera parte de la Ética, Spinoza establece que Dios no es causa transitiva, sino inmanente, lo cual hace que el orden tanto de Natura Naturans como de Natura Naturada sea necesario, y que por lo tanto Dios no actúe por voluntad, sino por necesidad. Al referirse al hombre, Spinoza vuelve a plantear que Dios no actúa por voluntad sino por necesidad, o, como lo dice en la proposición 17 de la primera parte, "Dios actúa según las leyes de su naturaleza". En esa medida en la segunda parte Spinoza sostiene que "Todo lo que está en potestad de Dios existe necesariamente" 10 .

Una conclusión muy importante para la discusión de su época, que se desprende de aquí, le permite señalar a Spinoza que, si no cabe pensar en la voluntad de Dios, por lo tanto tampoco cabe pensar en la posibilidad de la existencia de otros mundos. En este sentido no está de acuerdo con los mundos posibles y composibles de Leibniz, ya que sólo puede existir un mundo, aquel que brota con absoluta necesidad de la naturaleza misma de Dios. Es cierto que Leibniz, aunque trataba de salvar la libertad divina en el acto de la creación con la idea de infinitos mundos posibles, se veía obligado por la lógica de su pensamiento a señalar que el mundo en el que vivimos es el mejor de los mundos posibles, y por lo tanto el único que la voluntad divina podía elegir.

¿Pero qué relación tiene con el hombre esta vuelta al principio de operación necesaria del mundo? Pues que el ser humano, en tanto que modo del pensamiento y de la extensión, forma parte del orden necesario de la Natura Naturada, y eso implica que está sometido a una causalidad necesaria, no contingente. Esto hace que su acción no provenga tampoco de una voluntad absoluta, ajena a la causalidad, lo que plantea que no es el hombre aislado quien determina sus actos, sino que está regido por un sistema causal, y que ser consciente de las voliciones no implica producirlas de una forma absoluta. De tal manera que, cuando se supone que el hombre actúa guiado por una voluntad absoluta, se incurre en un error de comprensión, pues, como lo indica Spinoza en el Apéndice de la primera parte: "Los hombres opinan que son libres, porque son conscientes de sus voliciones y de su apetito, y ni por sueños piensan en las causa por las que están inclinados"11.

Ahora bien, teniendo en cuenta que los modos son independientes, Spinoza puede referirse al pensamiento y a la extensión por separado, y puesto que la voluntad pertenece al modo del atributo del pensamiento, es decir al alma, puede muy bien decir:

En el alma no se da ninguna voluntad absoluta o libre, sino que el alma es determinada a querer esto o aquello por una causa, que también es determinada por otra, ésta a su vez por otra, y así al infinito. ${ }^{12}$

\footnotetext{
9 EIIDef. 7.

10 EIIP3.

11 EI apéndice c.

12 EIIP48.
} 
La coherencia de la arquitectónica que construye Spinoza en la primera parte permite comprender, en la segunda, que existe un orden y conexión de las cosas, que es el mismo tanto en el pensamiento como en la extensión, y que ese orden es necesario. Sin embargo, no queda claro por qué Spinoza en el apéndice habla de voliciones en plural, que es aquello que tienen los hombres, y en la segunda parte habla sólo de voluntad, y más exactamente de voluntad absoluta, y niega que los hombres tengan voluntad absoluta, y por lo tanto tampoco libertad, si por libertad se entiende el ejercicio de esa voluntad absoluta.

En este sentido, Spinoza es muy meticuloso en su explicación. En el Escolio de la Proposición 48 de la Segunda Parte, acerca de la voluntad aclara que la formación de los universales a partir de los particulares no produce más que entes metafísicos, con lo cual se opone a cualquier consideración absoluta de las facultades del pensamiento, a saber, comprensiones universales de entender, desear, amar. Esta forma de pensar nos permite decir que la doctrina de Spinoza tiene un marcado nominalismo, a pesar de su no menos acendrado racionalismo: "De ahí que el entendimiento y la voluntad son a esta o a aquella idea o a esta o aquella volición, como es la petreidad a esta o aquella piedra, o como es hombre a Pedro y Pablo"13. Esta posición, aplicada al caso particular de la voluntad, le permite negar que los hombres tengan una voluntad absoluta, e incluso sostener que la voluntad entendida como una capacidad general para decidir no es más que una ficción o un ente metafísico, es decir, no real.

Sin embargo, Spinoza no se queda con una explicación negativa del concepto de voluntad. En el literal b del Escolio de la Proposición 48 explica que por voluntad entiende la capacidad para afirmar y negar, lo que significa identificar la facultad volitiva con la facultad cognoscitiva. Tesis que servirá para culminar la parte segunda de la Ética con la Proposición 49 y su corolario: "En el alma no se da ninguna volición, esto es, afirmación o negación, fuera de aquella que implica la idea en cuanto que es idea"14, puesto que "La voluntad y el entendimiento son una y la misma cosa"15

Ahora bien, aunque Spinoza establece que la fuente de las voliciones particulares, es decir, de las afirmaciones o negaciones, se encuentra en el plano del conocimiento ${ }^{16}$, no avanzaremos en el análisis de los diversos géneros de conocimiento, porque no lo consideramos para el propósito que nos guía. De esos géneros sólo rescatamos, para nuestro análisis de la volición, que una idea no es una imagen que se produzca por los sentidos, sino un concepto o una concepción del pensamiento ${ }^{17}$. Esto implica que afirmar o negar algo significa afirmar o negar un conocimiento, y a su vez ese conocimiento requiere otro anterior que lo sustente, tal como sería necesario tener el concepto de triángulo para poder afirmar que la suma de los ángulos interiores de un triángulo da ciento ochenta grados. Por lo tanto, no existe una voluntad absoluta, esto es una facultad general, sino voliciones particulares, que además son afirmaciones o negaciones de ideas, entendidas estas últimas como conceptos y sustentadas causalmente unas en otras.

13 EIIP48 escolio.

14 EIIP49.

15 EIIP48 corolario.

16 En la segunda parte Spinoza se ocupa de los grados de conocimiento, pero no nos detenemos en esa discusión. Sólo queremos anotar que Spinoza no asume nada de manera absoluta, sino gradual; en ese sentido el conocimiento tiene grados y, como lo veremos más adelante, la libertad también tiene grados.

17 Cfr. EIIP48 escolio. 
De esta manera se explica que pensar en el hombre como un ser libre debido a la autodeterminación de una voluntad absoluta, no sólo es un error, sino que en realidad no tiene sentido. Por lo tanto, la discusión clásica del Medioevo en torno al libre albedrio queda sin fundamento, puesto que en ella se parte del presupuesto de la inconmensurabilidad entre el entendimiento (razón) y la voluntad absoluta, al suponer, por un lado, un entendimiento finito y, por otro, una voluntad infinita de orden distinto a la razón.

Spinoza rechaza el presupuesto con tres razones: primera, no se puede concebir una voluntad absoluta, sino sólo voliciones particulares, luego la libertad que se desprende de la voluntad absoluta y que sustenta la cuestión del libre albedrío no es posible; segunda, las voliciones no son formas del deseo con las que el alma apetece o aborrece las cosas, sino de la facultad de afirmar o negar algo; y tercera el hombre se encuentra en un orden causal necesario. Por consiguiente se puede concluir, de acuerdo con el sistema de Spinoza, que el hombre no es libre en el sentido que solía usarse el término. Por eso, ante la pregunta acerca de la solución al dilema clásico medieval del asno de Buridano, Spinoza responde, dejando traslucir cierta molestia:

...digo que concedo sin reservas que un hombre puesto en tal equilibrio (a saber, que no percibe nada más que la sed, el hambre, tal comida y tal bebida, ambas a igual distancia de él), perecerá de hambre y de sed. ${ }^{18}$

De esta forma se abre el debate acerca de cómo hacer compatible el determinismo ${ }^{19}$ con la libertad, puesto que es claro que en Spinoza "el alma no puede ser causa libre de sus acciones" 20 . Sin embargo, como se indicó anteriormente, el sistema de Spinoza, aunque se opone a la concepción tradicional de libertad, ofrece otra concepción de libertad que se insinúa en la primera "contribución de la doctrina a la vida práctica"21 (Cfr. EII49escolio). De ella se puede colegir que la libertad para Spinoza tiene que ver con la aplicación del conocimiento a la vida práctica, de tal manera que el hombre por medio del conocimiento del orden divino o de Dios alcanza su máxima felicidad. Aprende a ignorar la fortuna en cuanto que comprende que todo (prosperidad y adversidad) se sigue con la misma necesidad y en ese mismo sentido "a no odiar a nadie, ni despreciar, ni burlarse, ni irritarse, ni envidiar a nadie" 22 .

18 EIIP49 escolio k.

19 Smith en su libro retoma un comentario de Isahiah Berlin sobre el determinismo, que nos recuerda la antropología forense de finales del siglo XIX y principio del siglo XX, que pretendía determinar el comportamiento criminal por el tamaño y la forma de la cabeza de los individuos, o algunas teorías de genética, mucho más recientes, que observan un gen determinado en las personas de comportamiento agresivo. Smith por su parte comenta: "La idea de Berlín no es que el determinismo adoptado por Spinoza sea falso. Si el determinismo fuera simplemente falso, no sería incompatible con la libertad; sería simplemente irrelevante. Berlín, como otros incompatibilistas, en alguna ocasión concede incluso que, con el avance de las ciencias empíricas, una ciencia predictiva del comportamiento humano no es algo descartable. Su principal preocupación es la de que si se llegase a tal ciencia, no sólo nos negaría el poder de auto-iniciación de nuestras acciones, sino que se nos desposeería del vocabulario moral de elogio y censura, culpa y responsabilidad". Spinoza y el libro de la vida. Op. Cit. p. 113.

20 EIIP48 Demostración.

21 Las contribuciones conforman la última parte de la segunda parte de la ética, y en ellas Spinoza responde objeciones y plantea los aportes de su doctrina.

22 EIIP49 escolio 1,3 
No obstante, para poder comprender con mayor claridad y en sentido afirmativo el concepto de libertad en Spinoza, tenemos que recurrir a las partes tercera y cuarta de la Ética. Por ahora vale la pena destacar que, de la misma manera como lo hizo con el Apéndice de la primera parte, Spinoza termina la segunda parte de la Ética estableciendo una conexión con la política, al señalar que su doctrina no sólo está orientada a la práctica personal, sino que también se dirige a la vida en sociedad:

Finalmente esta doctrina ayuda también no poco a la sociedad común, en cuanto que enseña de qué forma deben ser gobernados y dirigidos los ciudadanos, a saber, no para que presten un servicio, sino para que hagan libremente lo que es mejor ${ }^{23}$.

Como lo explica Amalia Boyer: "la política es inseparable de la ontología. Por ende, toda ontología es política y toda política es en sí misma una ontología. La relación recíproca entre ontología y política puede ser identificada como la cuestión de su 'paralelismo'. El paralelismo de lo ontológico y lo político encuentra una primera formulación en el pensamiento de Spinoza. Este sólo puede escribir una ética y una política con base a su análisis de la sustancia"24.

Otra característica de esta nueva concepción del cuerpo permite comprender la singularidad de cada hombre por el cuerpo, en este sentido se puede entender el cuerpo como el fundamento de la diferencia entre los hombres. Al respecto Spinoza sostiene que la singularidad está dada por la finitud y la existencia limitada de los cuerpos. Sin embargo, Spinoza también propone un principio de unión en todos los cuerpos, de acuerdo con el cual si varios cuerpos se unen con el mismo fin, esta conformidad de cuerpos hace que se los pueda entender como una cosa singular, como un solo cuerpo. El caso por excelencia de conformidad en el que varios cuerpos actúan como un solo cuerpo se presentaría en la política donde los cuerpos de los ciudadanos se unen con el fin de instituir el cuerpo político, y desde el atributo cogitans Spinoza insiste en los dos tratados que los hombres en tanto que sujetos políticos obren "como una sola mente".

También encontramos que la cuestión para Spinoza reside en explicar cómo se produce esa unión de cuerpos para que devenga política, puesto que no todo encuentro de cuerpos es político. De hecho, los cuerpos se encuentran constantemente y muchos de esos encuentros se dan al azar lo que hace que los hombres padezcan afectos alegres o tristes sin el menor control entonces la pregunta que intentamos esclarecer es ¿qué se necesita para superar ese estado de sometimiento del hombre al azar de los encuentros para devenir, en efecto, sujeto político?

La respuesta va en orden a la concomitancia entre cuerpo y mente, en cuanto la mente empieza a conocer las nociones comunes concomitantes al encuentro de dos cuerpos, es decir, en cuanto conoce qué es lo común a los dos cuerpos, y por lo tanto qué causalidad se produce entre los dos, entonces el hombre comienza a liberarse. Esta relación, que es lo políticamente más importante del reflexión en torno al cuerpo de Spinoza, libera al hom-

23 EIIP49 escolio, $1,4$.

24 Boyer, A., Materialismo Ontológico y Política en Spinoza Deleuze y Guatari. En: Eidos, Universidad del Norte, No 1. 2003 pgs. 94-106. (Conferencia pronunciada en el marco del primer congreso de Ética y Filosofía Política en Alcalá de Henares, España, Septiembre de 2002). 
bre del sometimiento al padecer de los encuentros y hace que el hombre, desde su cuerpo, empiece a ejercer, efectivamente, una acción sobre lo que siente o en otras palabras adquiera libertad: "digo que nosotros actuamos cuando en nosotros o fuera de nosotros se produce algo de lo que somos causa adecuada" 25 .

Así pues, un encuentro entre cuerpos para que sea lugar de libertad tiene que darse en tanto que un cuerpo sea causa adecuada, lo que implica, en el modo cuerpo, establecer lo común con el cuerpo afectante o afectado, y en el modo mente, establecer, por medio del entendimiento racional y reflexivo, la conexión entre las ideas de los respectivos cuerpos. Cuando así se procede, de acuerdo con la explicación de Spinoza, configuramos ideas adecuadas, y estas implican que actuamos y no padecemos, y por consiguiente que somos libres, y el Estado sería el mejor lugar para alcanzar la libertad.

Finalmente, estas relaciones, como se explica en la primera parte, constituyen el principio de diferencia entre cuerpos, pero también lo que les permite a los cuerpos convenir o disconvenir, de donde se deriva una composición o descomposición de los cuerpos. En el primer caso, de conveniencia, se produce un encuentro de composición en el que cada cuerpo obtiene beneficiado, puesto que los cuerpos aumentan sus relaciones. En el segundo caso se presenta un encuentro de descomposición, esto es, un encuentro en el que las relaciones disconvienen de tal manera que uno de los cuerpos divide las partes del otro y trasforma una de sus relaciones en contraria a su propio cuerpo, provocando que éste se "descomponga" en dos naturalezas.

En fin, Steven Smith considera que esta reflexión en torno a la política nos permite comprender que Spinoza tiene una concepción más rica de sentido que la mera explicación natural, y que por lo tanto podemos concluir que: "la vida [que en sentido material para Spinoza es el cuerpo] no es una mera categoría biológica, sino un imperativo moral" 26 .

\section{Bibliografía}

Bennett, Jonathan (1990): Un Estudio de la Ética de Spinoza, Fondo de Cultura Económica, México.

Boyer, Amalia (2003): Materialismo Ontológico y Política en Spinoza Deleuze y Guatari», Eidos (Universidad del Norte), No 1. pp. 94-106.

Chauí, Marilena (2004): Política en Spinoza, Gorla, Argentina.

Deleuze, Gilles (1975): Spinoza y el problema de la expresión, Muchnik, España.

Deleuze, Gilles (2001): Spinoza: Filosofía práctica, Tusquets, Barcelona

Fernández, Eugenio (1996): Articulación crítica de Ontología y Política en B. Spinoza, Revista de Filosofía, Num. 15, Chile, pp. 97-126.

Garret, Don (1986): A free man always acts honestly, not deceptively en Spinoza: Issues and Directions, The proceedings of the Chicago Conference, editado por Edwin Curley y Pierre-Francois Moureau, Brill, Chicago, pp. 221-238.

Garrido, Juan (1992): Comentario Ético-Político a la afirmación spinocista: Homini nihil inter res singulares utilius datur quam homo en Ética de Spinoza. Fundamentos y Significado, Universidad de Castilla-La Mancha, Castilla-La Mancha.

25 EIII Definción2.

26 Spinoza y el libro de la vida Op. Cit. p. 115 
Gueroult, Martial (1968): Spinoza 1, Aubier Montaigne, Paris.

Lloyd, Genevive (1996): Spinoza, Routledge, New York.

Hubeling, Hubertus (1981): Spinoza, Herder, Barcelona.

Mason, Richard (1997): The God of Spinoza. A philosophical study, Cambridge, U.K.

Matheron, André (1969): Individu et Communauté chez Spinoza, Les Editions de Minuit, Paris.

Smith, Steven (2007): Spinoza y el libro de la vida. Libertad y Redención en la Ética, Biblioteca Nueva, Madrid.

Spinoza, Baruch (2005): Ética demostrada según el orden geométrico, Trotta, Madrid.

Spinoza, Baruch (1990): Tratado Breve, Alianza, Madrid.

Spinoza, Baruch (1986): Tratado Político, Alianza, Madrid.

Spinoza, Barcuh (2003): Tratado Teológico-Político, Alianza, Madrid.

Spinoza, Baruch (1994): Traité des autorités théologique et politique, Gallimard, Paris.

Genevive, Lloyd (ed) (2001): Spinoza, Critical Assessments of leading philosophers, Vol I a Vol IV, Routledge; New York.

Tatían, Diego (2001): La Cautela del Salvaje; pasiones y política en Spinoza, Adriana Hidalgo, Buenos Aires.

Yovel, Yirmiyahu (2001): Spinoza: the psychology of the Multitude and the Uses of Language en Spinoza: Critical Assessments of leading philosophers vol III, Routledge, New York. 
